\title{
Measurements of intracrater flow dynamics utilizing a mound-bearing crater in a refractive index matched environment
}

\author{
D. G. Gundersen ${ }^{1}$, K. T. Christensen ${ }^{1,2}$, G. Blois ${ }^{1}$ \\ ${ }^{1}$ University of Notre Dame, Department of Aerospace and Mechanical Engineering, Notre Dame, USA \\ ${ }^{2}$ Illinois Institute of Technology, Depts. of Mechanical, Materials \& Aerospace Engineering and Civil, \\ Architectural \& Environmental Engineering, Chicago, USA
}

\section{Introduction}

The processes controlling crater mound formation are the subject of ongoing research (Bennett and Bell III, 2016). Several theories exist on the formation of a central mound, with those pointing to wind processes as the predominant driving mechanisms being among the most compelling (Kite et al., 2013; Day et al., 2016; Anderson and Day, 2017). Few experimental studies have been conducted to uncover impact crater driven flow dynamics. As such, direct and experimental flow measurements that could be used to validate previously developed fluid-topography interaction theories are not yet available. The objective of this study is to elucidate the intra- and extra-crater circulation induced by unidirectional winds using experimentation on scaled models coupled with high spatial and temporal resolution flow measurements.

\section{Experiments}

PIV measurements were performed on both an idealized crater shape and a model sourced from a digital elevation map (DEM) of Gale Crater. Laboratory experiments were performed on these physical models in the Refractive Index-Matched (RIM) flow facility at Notre Dame leveraging an aqueous solution of sodium iodide (NaI) as the working fluid and cast acrylic crater models. The RIM technique renders the solid model effectively invisible and thus allows unobstructed and unaberrated optical access to all regions of the flow produced by the crater. Slight optical mismatches between the solid and fluid RIs were minimized by fine tuning the fluid temperature.

The models included both a synthetic crater shape based upon a mathematical model that served as a proxy of real craters, and a digital elevation map (DEM) of Gale Crater, sourced from the HRSC experiment on Mars Express (Jaumann et al., 2007). A low-pass filter was applied to the DEM and the vertical scale was exaggerated by a factor of three in order to adhere to constraints of the facility. Planar particle image velocimetry (PIV) measurements were performed at two streamwise-wall-normal $(x-y)$ plane positions and three wall-parallel $(x-z)$ planes. Measurements were also performed at four freestream velocities for each measurement plane to examine potential Reynolds number effects, yielding a total of 20 independent datasets.

\section{Results}

Statistical analysis was performed in order to reconstruct the spatial behavior of the underlying turbulence. Here, an example of results is included in order to provide an overview of the measurements obtained in this study. Figure 11 presents contours of the streamwise and wall-normal Reynolds normal stresses, normalized by the square of the freestream velocity $U_{\infty}$, in both of the streamwise-wall-normal measurement planes. The measurement plane corresponding to Fig. 1 $1(\mathrm{a}, \mathrm{c})$ is positioned at the spanwise plane of symmetry, while in Fig. 1 $(\mathrm{b}, \mathrm{d})$ the measurement plane is offset in the spanwise direction by $1.3 H$, where $H$ is the crater rim height. The contrast between the flow maps of the turbulent stresses in the two different planes provides a qualitative measure of the three-dimensionality of the flow.

A shear layer forms at the upstream rim of the crater and undergoes a series of shear-obstacle interactions. In the centerline plane (Fig. 1 $1(\mathrm{a}, \mathrm{c}, \mathrm{e})$ ) the shear layer interacts with the mound, forming a merged shear layer that then impinges into the interior of the downstream rim. Vertical profiles of turbulent stresses in the crater wake show imprints from the upstream shear layer dynamics, as they contain two maxima in turbulent 

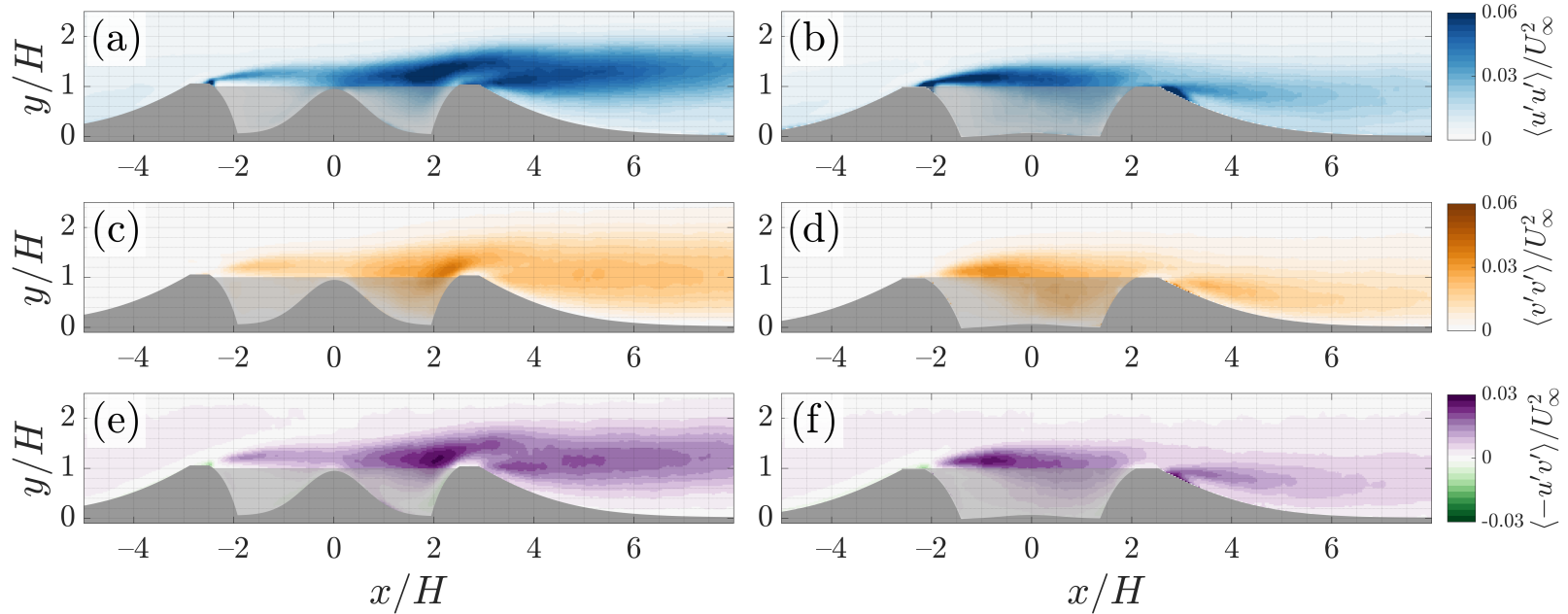

Figure 1: Contours of normalized (a,b) streamwise turbulence stress, $\left\langle u^{\prime} u^{\prime}\right\rangle$, (c,d) wall-normal turbulence stress, $\left\langle v^{\prime} v^{\prime}\right\rangle$, and (e,f) turbulence shear stress, $\left\langle-u^{\prime} v^{\prime}\right\rangle$, in the wall-normal centerline plane (left) and the spanwise offset wall-normal plane (right).

stresses. Measurements show regions of elevated turbulence in the intracrater space below the rim, particularly for the offset plane (Fig. 1 1 ,d,f). The shear layers, populated by energetic coherent structures, are presumably linked to elevated transport of sediment and could account for some of the erosion/deposition processes hypothesized by previous studies.

Experiments on the Gale Crater model highlight similar areas of elevated turbulent stresses. These measurements could be used to support observational studies based on the interpretation of surface features in areas targeted by current missions. Details of this study, published in Gundersen et al. (2021), will be provided in the presentation.

\section{Conclusions}

The results presented herein demonstrate the effectiveness of the RIM technique to image the flow within complex topographies, as well as some of its limitations. The measurements near the surface and within the intracrater space would not have been possible without utilizing a RIM approach. Experiments were performed on a Gale Crater model in addition to the idealized crater model. The results for the Gale Crater model shows flow features similar to the results on the idealized model. This gives evidence that the idealized geometry can be used as an analogue to geometries based on real impact craters.

\section{References}

Anderson W and Day M (2017) Turbulent flow over craters on mars: Vorticity dynamics reveal aeolian excavation mechanism. Phys Rev E 96:76-128

Bennett KA and Bell III JF (2016) A global survey of martian central mounds: Central mounds as remnants of previously more extensive large-scale sedimentary deposits. Icarus 264:331-341

Day M, Anderson W, Kocurek G, and Mohrig D (2016) Carving intracrater layered deposits with wind on mars. Geophys Res Lett 43:2473-2479

Gundersen D, Blois G, and Christensen KT (2021) Flow past mound-bearing impact craters: An experimental study. Fluids 6:216

Jaumann R, Neukum G, Behnke T, Duxbury TC, Eichentopf K, Flohrer J, Gasselt S, Giese B, Gwinner K, Hauber E et al. (2007) The high-resolution stereo camera (hrsc) experiment on mars express: Instrument aspects and experiment conduct from interplanetary cruise through the nominal mission. Planet Space Sci 55:928-952

Kite ES, Lewis KW, Lamb MP, Newman CE, and Richardson MI (2013) Growth and form of the mound in gale crater, mars: Slope wind enhanced erosion and transport. Geology 41:543-546 